\title{
Mean-square heterogeneous synchronization of interdependent networks with stochastic disturbances
}

\author{
Tianjiao Guo ${ }^{1,2}$, Lilan Tu $^{1,2^{*}}$ and Jiabo Chen ${ }^{1,2}$
}

\author{
${ }^{*}$ Correspondence: \\ tulilan@wust.edu.cn \\ ${ }^{1}$ Hubei Province Key Laboratory of \\ Systems Science in Metallurgical \\ Process, Wuhan University of \\ Science and Technology, Wuhan, \\ China \\ ${ }^{2}$ College of Science, Wuhan \\ University of Science and \\ Technology, Wuhan, China
}

\begin{abstract}
In this paper, the mean-square asymptotical heterogeneous synchronization of interdependent networks with stochastic disturbances, which is a zero-mean real Wiener process, is investigated. The network discussed consists of two sub-networks, which are one-by-one inter-coupled. The unknown but bounded nonlinear coupling functions not only exist in the intra-coupling but also in the inter-coupling between two sub-networks. Based on the stochastic Lyapunov stability theory, adaptive control, Itô formula and the linear matrix inequality, several sufficient conditions are proposed to guarantee adaptive mean-square heterogeneous asymptotical synchronization of the interdependent networks. In order to better illustrate the feasibility and effectiveness of the synchronization conditions derived in this brief, numerical simulations are provided finally.
\end{abstract}

Keywords: Interdependent networks; Stochastic disturbances; Mean-square heterogeneous asymptotical synchronization; Itô formula

\section{Introduction}

Interdependent networks are a special form of complex networks which are coupled by two or more sub-networks. With the progress of science and technology, there are more and more dependencies in different infrastructures or systems. Most of the modern society infrastructures or systems are interdependent networks, such as electric-calculator networks [1], traffic-transportation networks [2], and so on. A typical example for interdependent networks is the Italian electrical destruction on Sept. 28th, 2003. The shutdown of power stations directly led to the failure of nodes in the computer networks, which in turn caused deeper breakdown of power stations $[1,3]$. Buldyrev et al. firstly proposed the interdependent networks in 2010, which opened the new chapter of complex networks [4]. They reveal that the failure of a node in one sub-network may cause the failure of interdependent nodes in other sub-networks. The investigation and growing understanding of the interdependent networks will enable us to make the infrastructures we use in everyday life more efficient and more robust. From then on, the investigation of interdependent networks became an issue of focus in the network science [5].

Recently, there have been some results for interdependent networks [6-12]. In Ref. [6], Zhang et al. extended the system's robustness under different attack strategies. In Ref.

(c) The Author(s) 2019. This article is distributed under the terms of the Creative Commons Attribution 4.0 International License (http://creativecommons.org/licenses/by/4.0/), which permits unrestricted use, distribution, and reproduction in any medium, provided you give appropriate credit to the original author(s) and the source, provide a link to the Creative Commons license, and indicate if changes were made. 
[7], Ji et al. improved the robustness of interdependent networks by adding connectivity. In Ref. [8], Xu et al. studied the generalized mutual synchronization between two controlled interdependent networks. In Ref. [9], the authors explored the local adaptive heterogeneous synchronization for interdependent networks with delayed coupling consisting of two sub-networks. In Ref. [10], the author proposed new methods for improving the robustness of interdependent networks. In Ref. [12], the author studied the coevolution of strategy and network interdependence. When investigating interdependent networks, stochastic disturbances are inevitable and should be considered. They exist everywhere [13-15]. Interdependent networks usually are affected by stochastic disturbances. For example, information transmission is a noisy process, which may affect the accuracy of messages. However, until now, there has been little research on interdependent networks with stochastic perturbations.

Synchronization is an interesting and meaningful issue in the research of complex networks. The investigation of synchronization for dynamical systems was initiated in the early 1980s for deterministic chaos. At present, many scholars have explored the synchronization of complex networks [16-18]. Due to the disturbances of networks, there have been many works in the field of synchronization for complex networks with stochastic disturbances [19-25]. In Ref. [19], the finite-time stochastic synchronization of time-delay neural networks with noise was investigated. In Ref. [23], the author studied the finite/fixed-time pinning synchronization of complex networks with stochastic disturbances. In Ref. [24], the synchronization between each node in the drive layer and its counterpart in the response layer of this sort of duplex networks with delayed nodes and noise perturbation is explored. Though there is increasing research relating to the synchronization of interdependent networks $[8,9,26]$, few people have explored the synchronization problem of interdependent networks with stochastic disturbances.

Motivated by the above-mentioned issues, in this paper, we discuss the synchronization of interdependent networks with stochastic disturbances. The whole of the networks consists of two sub-networks with "one-by-one" interdependence. Based on the stochastic Lyapunov stability theory, adaptive control, the Itô formula and the linear matrix inequality, some control schemes related to mean-square heterogeneous synchronization are obtained in this paper. In the end, some simulations verify the feasibility and effectiveness of our conclusions.

\section{Preliminaries}

Considering interdependent networks which have two sub-networks $G^{1}$ and $G^{2}$, each subnetwork consisting of $N$ identical dynamical nodes with nonlinear couplings and stochastic disturbances. It can be characterized by

$$
\begin{aligned}
& d\left(x_{i}^{k}(t)\right)= {\left[f^{k}\left(x_{i}^{k}(t)\right)+d^{k} \sum_{j=1}^{N} A_{i j}^{k} H^{k}\left(x_{j}^{k}(t)\right)+\sum_{l=1}^{2} c^{k l} H^{k}\left(x_{i}^{l}(t)\right)\right] d t } \\
&+B^{k}\left(t, x_{i}^{k}(t)-s^{k}(t)\right) d W(t) \\
& i=1, \ldots, N, k=1,2, l=1,2
\end{aligned}
$$

where $x_{i}^{k}=\left(x_{i 1}^{k}, x_{i 2}^{k}, \ldots, x_{i n}^{k}\right) \in R^{n}$ are the state variables of the $i$ th node in the $k$ th subnetwork, $f^{k}: R^{n} \rightarrow R^{n}$ is a continuous but bounded nonlinear function, $d^{k}$ is the intra- 
coupling strength of the $k$ th sub-network, $c^{k l}$ is the inter-coupling strength between the $k$ th sub-network and the $l$ th sub-network, it satisfying

$$
c^{11}=c^{22}=-c \text { and } c^{12}=c^{21}=c .
$$

$A^{k}=\left(A_{i j}^{k}\right)_{N \times N}$ is the intra-coupling configuration matrix of the $k$ th sub-network. If there exists a connection from the $i$ th node to the $j$ th node in the $k$ th sub-network, then $A_{i j}^{k}=$ $A_{j i}^{k}=1$, otherwise, $A_{i j}^{k}=0$. At the same time, $A^{k}$ satisfying

$$
A_{i i}^{k}=-\sum_{\substack{j=1 \\ j \neq i}}^{N} A_{i j}^{k}=-\sum_{\substack{j=1 \\ j \neq i}}^{N} A_{j i}^{k}, \quad i=1,2, \ldots, N .
$$

$H^{k}: R^{n} \rightarrow R^{n}$ is the coupling function of the $k$ th sub-network, $B^{k}$ is a nonlinear and bounded function, and $W(t)$ is a zero-mean real Wiener process satisfying

$$
E(d W(t))=0, \quad E\left((d W(t))^{\mathrm{T}}(d W(t))\right)=d t .
$$

Adding suitable controllers $u_{i}^{k}(t)$ to the interdependent networks (1), we get

$$
\begin{aligned}
d\left(x_{i}^{k}(t)\right)= & {\left[f^{k}\left(x_{i}^{k}(t)\right)+d^{k} \sum_{j=1}^{N} A_{i j}^{k} H^{k}\left(x_{j}^{k}(t)\right)+\sum_{l=1}^{2} c^{k l} H^{k}\left(x_{i}^{l}(t)\right)+u_{i}^{k}(t)\right] d t } \\
& +B^{k}\left(t, x_{i}^{k}(t)-s^{k}(t)\right) d W(t), \\
k=1,2 ; l=1,2 . &
\end{aligned}
$$

Definition 1 (Mean-square heterogeneous asymptotical synchronization) The interdependent networks (1) are said to achieve mean-square heterogeneous asymptotical synchronization if

$$
\lim _{t \rightarrow \infty} E\left(\left\|x_{i}^{k}(t)-s^{k}(t)\right\|^{2}\right)=0, \quad i=1,2, \ldots, N, k=1,2
$$

where $s^{k}(t) \in R^{n}(k=1,2)$ is a solution of an isolated node of the $k$ th sub-network, namely

$$
\dot{s}^{k}(t)=f^{k}\left(s^{k}(t)\right), \quad k=1,2 .
$$

Equation (6) can be rewritten as

$$
\begin{aligned}
& \dot{s}^{k}(t)=f^{k}\left(s^{k}(t)\right)+d^{k} \sum_{j=1}^{N} A_{i j}^{k} H^{k}\left(s^{k}(t)\right)+\sum_{l=1}^{2} c^{k l}\left(H^{k}\left(s^{l}(t)\right)-H^{k}\left(s^{l}(t)\right)\right), \\
& \quad k=1,2, l=1,2 .
\end{aligned}
$$

Remark 1 In this paper, we mainly discuss the mean-square heterogeneous asymptotical synchronization of interdependent networks (4) under the controllers $u_{i}^{k}(t)$. 
In order to better illustrate the results of this paper, the following assumptions and lemmas are introduced.

Assumption 1 Suppose that there exists a constant matrix $L_{r}$ with appropriate dimensions such that

$$
\left\|f\left(x_{1}(t)\right)-f\left(x_{2}(t)\right)\right\| \leq\left\|L_{r}\left(x_{1}(t)-x_{2}(t)\right)\right\|, \quad r=0,1,
$$

for $\forall x_{1}(t), x_{2}(t) \in R^{n}$.

Assumption 2 Assume that $\hat{H}(t)=D H^{1}\left(s^{1}(t)\right)=\left(\hat{h}_{i j}(t)\right)_{n \times n} \in R^{n \times n}$ is the Jacobian matrix of $H^{1}\left(x_{i}^{1}(t)\right)$ at $s^{1}(t)$, and denote $\hat{H}=\left(\hat{h}_{i j}\right)_{n \times n} \in R^{n \times n}$, where $\hat{h}_{i j}$ is the maximum value of $\hat{h}_{i j}(t)$. Similarly, $\tilde{H}(t)=D H^{2}\left(s^{2}(t)\right)=\left(\tilde{h}_{i j}(t)\right)_{n \times n} \in R^{n \times n}$ is the Jacobian matrix of $H^{2}\left(x_{i}^{2}(t)\right)$ at $s^{2}(t)$, and let $\tilde{H}=\left(\tilde{h}_{i j}\right)_{n \times n} \in R^{n \times n}$, where $\tilde{h}_{i j}$ is the maximum value of $\tilde{h}_{i j}(t)$.

Assumption 3 Suppose that there exists a constant matrix $L_{r}$ with suitable dimensions such that

$$
B(t, x(t)-y(t)) \leq\left\|L_{r}(x(t)-y(t))\right\|, \quad r=2,3,
$$

for all $x(t), y(t) \in R^{n}$.

Let the synchronization error be $e_{i}^{k}(t)=x_{i}^{k}(t)-s^{k}(t)(i=1,2, \ldots, N, k=1,2)$. Then the error system can be described as

$$
\left\{\begin{aligned}
d\left(e_{i}^{1}(t)\right)= & {\left[\left(f^{1}\left(x_{i}^{1}(t)\right)-f^{1}\left(s^{1}(t)\right)\right)\right.} \\
& +d^{1} \sum_{j=1}^{N} A_{i j}^{1} \hat{H}(t) e_{j}^{1}(t)+c H^{1}\left(s^{2}(t)\right)-c H^{1}\left(s^{1}(t)\right) \\
& \left.+\sum_{l=1}^{2} c^{1 l} \hat{H}(t) e_{i}^{l}(t)+u_{i}^{1}(t)\right] d t+B^{1}\left(t, e_{i}^{1}(t)\right) d W(t), \\
d\left(e_{i}^{2}(t)\right)= & {\left[\left(f^{2}\left(x_{i}^{2}(t)\right)-f^{2}\left(s^{2}(t)\right)\right)\right.} \\
& +d^{2} \sum_{j=1}^{N} A_{i j}^{2} \tilde{H}(t) e_{j}^{2}(t)+c H^{2}\left(s^{1}(t)\right)-c H^{2}\left(s^{2}(t)\right) \\
& \left.+\sum_{l=1}^{2} c^{2 l} \tilde{H}(t) e_{i}^{l}(t)+u_{i}^{2}(t)\right] d t+B^{2}\left(t, e_{i}^{2}(t)\right) d W(t) .
\end{aligned}\right.
$$

Remark 2 The mean-square heterogeneous asymptotical synchronization for interdependent networks (1) also can be obtained if

$$
\lim _{t \rightarrow \infty} E\left(\left\|e_{i}^{k}(t)\right\|^{2}\right)=0
$$

Therefore, in the following, we mainly discuss the mean-square heterogeneous asymptotical stability at the origin for the system (8).

Remark 3 Let $I$ represent the identity matrix with the appropriate dimensions.

Lemma 1 ([27]) For any matrices $X, Y \in R^{n \times n}$, the following matrix inequality holds:

$$
X^{\mathrm{T}} Y+Y^{\mathrm{T}} X \leq X^{\mathrm{T}} A X+Y^{\mathrm{T}} A^{-1} Y
$$

in which $A^{\mathrm{T}}=A>0, A \in R^{n \times n}$. 
Lemma 2 (Schur complement [28]) Assume $Q(x)=Q(x)^{\mathrm{T}}, R(x)=R(x)^{\mathrm{T}}$ and $S(x)$ all the functions of $x$, the following linear matrix inequality:

$$
\left[\begin{array}{cc}
Q(x) & S(x) \\
S(x)^{\mathrm{T}} & R(x)
\end{array}\right]<0
$$

is equivalent to any of the following conditions:

(1) $R(x)<0, Q(x)-S(x) R(x)^{-1} S(x)^{\mathrm{T}}<0$,

(2) $Q(x)<0, R(x)-S(x)^{\mathrm{T}} Q(x)^{-1} S(x)<0$.

\section{Main results}

In this section, according to stochastic Lyapunov stability theory, adaptive control method and the Itô formula, combined with the linear matrix inequality, with suitable adaptive controllers, adaptive mean-square heterogeneous asymptotical synchronization of the interdependent networks (1) is investigated.

Theorem 1 Suppose that Assumptions 1-3 hold, and there exist two positive matrices $P$ and $Q$, such that

$$
\Lambda=\left[\begin{array}{cccc}
\tilde{T}_{i}^{1} & \Phi & \Theta^{1} & 0 \\
\Phi^{\mathrm{T}} & \tilde{T}_{i}^{2} & 0 & \Theta^{2} \\
\Theta^{1^{\mathrm{T}}} & 0 & \Psi^{1} & 0 \\
0 & \Theta^{2^{\mathrm{T}}} & 0 & \Psi^{2}
\end{array}\right]<0
$$

where

$$
\begin{aligned}
& \tilde{T}_{i}^{1}=\lambda^{1} L_{0}^{\mathrm{T}} L_{0}+P+d^{1} \sum_{j=1}^{N}\left|A_{j i}^{1}\right| I+\lambda^{1} L_{2}^{\mathrm{T}} L_{2}-c \hat{H}^{\mathrm{T}} P-c P \hat{H}-2 d P, \\
& \tilde{T}_{i}^{2}=\lambda^{2} L_{1}^{\mathrm{T}} L_{1}+Q+d^{2} \sum_{j=1}^{N}\left|A_{j i}^{2}\right| I+\lambda^{2} L_{3}^{\mathrm{T}} L_{3}-c \tilde{H}^{\mathrm{T}} Q-c Q \tilde{H}-2 d Q, \\
& \Phi=c P \hat{H}+c \tilde{H}^{\mathrm{T}} Q, \quad \Theta^{1}=P \hat{H}, \quad \Theta^{2}=Q \tilde{H}, \\
& \Psi^{1}=\frac{-1}{d^{1} \sum_{j=1}^{N}\left|A_{i j}^{1}\right|} I, \quad \Psi^{2}=\frac{-1}{d^{2} \sum_{j=1}^{N}\left|A_{i j}^{2}\right|} I,
\end{aligned}
$$

in which $L_{0}, L_{1}, L_{2}$ and $L_{3}$ are constant matrices with suitable dimensions, $\lambda^{1}$ and $\lambda^{2}$ are the maximum eigenvalues of the matrix $P$ and $Q$, respectively, then the interdependent networks (1) can obtain adaptive mean-square heterogeneous asymptotical synchronization under the control law

$$
\left\{\begin{array}{l}
u_{i}^{1}(t)=c H^{1}\left(s^{1}(t)\right)-c H^{1}\left(s^{2}(t)\right)-d_{i}^{1} e_{i}^{1}(t), \\
u_{i}^{2}(t)=c H^{2}\left(s^{2}(t)\right)-c H^{2}\left(s^{1}(t)\right)-d_{i}^{2} e_{i}^{2}(t),
\end{array}\right.
$$


and the adaptive law

$$
\left\{\begin{array}{l}
\dot{d}_{i}^{1}=k_{i}^{1} e_{i}^{1}(t)^{\mathrm{T}} P e_{i}^{1}(t) \\
\dot{d}_{i}^{2}=k_{i}^{2} e_{i}^{2}(t)^{\mathrm{T}} Q e_{i}^{2}(t)
\end{array}\right.
$$

where $d$ is the estimation of $d_{i}^{1}$ and $d_{i}^{2} . k_{i}^{1}$ and $k_{i}^{2}$ are the feedback gains of sub-networks $G^{1}$ and $G^{2}$.

Proof According to control law (11) and adaptive law (12), we can rewrite the error system (8) in the following form:

$$
\left\{\begin{aligned}
d\left(e_{i}^{1}(t)\right)= & {\left[\left(f^{1}\left(x_{i}^{1}(t)\right)-f^{1}\left(s^{1}(t)\right)\right)\right.} \\
& \left.+d^{1} \sum_{j=1}^{N} A_{i j}^{1} \hat{H}(t) e_{j}^{1}(t)+\sum_{l=1}^{2} c^{1 l} \hat{H}(t) e_{i}^{l}(t)-d_{i}^{1} e_{i}^{1}(t)\right] d t \\
& +B^{1}\left(t, e_{i}^{1}(t)\right) d W(t), \\
d\left(e_{i}^{2}(t)\right)= & {\left[f^{2}\left(x_{i}^{2}(t)\right)-f^{2}\left(s^{2}(t)\right)\right) } \\
& +d^{2} \sum_{j=1}^{N} A_{i j}^{2} \tilde{H}(t) e_{j}^{2}(t) \\
& \left.+\sum_{l=1}^{2} c^{2 l} \tilde{H}(t) e_{i}^{l}(t)-d_{i}^{2} e_{i}^{2}(t)\right] d t+B^{2}\left(t, e_{i}^{2}(t)\right) d W(t) .
\end{aligned}\right.
$$

Construct the following Lyapunov function:

$$
V(t)=\sum_{i=1}^{N}\left[e_{i}^{1}(t)^{\mathrm{T}} P e_{i}^{1}(t)+e_{i}^{2}(t)^{\mathrm{T}} Q e_{i}^{2}(t)+\frac{\left(d_{i}^{1}-d\right)^{2}}{k_{i}^{1}}+\frac{\left(d_{i}^{2}-d\right)^{2}}{k_{i}^{2}}\right] .
$$

Then with the Itô formula, the derivative of $V(t)$ on the system (13) is as follows:

$$
\begin{aligned}
d V(t)= & \sum_{i=1}^{N}\left\{d e_{i}^{1}(t)^{\mathrm{T}} P e_{i}^{1}(t)+e_{i}^{1}(t)^{\mathrm{T}} P d e_{i}^{1}(t)+d\left[\frac{\left(d_{i}^{1}-d\right)^{2}}{k_{i}^{1}}\right]\right. \\
& \left.+d e_{i}^{2}(t)^{\mathrm{T}} Q e_{i}^{2}(t)+e_{i}^{2}(t)^{\mathrm{T}} Q d e_{i}^{2}(t)+d\left[\frac{\left(d_{i}^{2}-d\right)^{2}}{k_{i}^{2}}\right]\right\} \\
= & \sum_{i=1}^{N}\left\{\left[\left(f^{1}\left(x_{i}^{1}(t)\right)-f^{1}\left(s^{1}(t)\right)\right)^{\mathrm{T}} P e_{i}^{1}(t)\right.\right. \\
& +d^{1} \sum_{j=1}^{N} A_{i j}^{1} e_{j}^{1}(t)^{\mathrm{T}} \hat{H}(t)^{\mathrm{T}} P e_{i}^{1}(t)+e_{i}^{1}(t)^{\mathrm{T}} P\left(f^{1}\left(x_{i}^{1}(t)\right)-f^{1}\left(s^{1}(t)\right)\right) \\
& \left.+d^{1} \sum_{j=1}^{N} A_{i j}^{1} e_{i}^{1}(t)^{\mathrm{T}} P \hat{H}(t) e_{j}^{1}(t)\right] d t \\
& +\left[\left(f^{2}\left(x_{i}^{2}(t)\right)-f^{2}\left(s^{2}(t)\right)\right)^{\mathrm{T}} Q e_{i}^{2}(t)\right. \\
& \left.\left.+d^{2} \sum_{j=1}^{N} A_{i j}^{2} e_{i}^{2}(t)^{\mathrm{T}} Q \tilde{H}(t) e_{j}^{2}(t)\right] d t\right\} \\
& +d^{2} \sum_{j=1}^{N} A_{i j}^{2} e_{j}^{2}(t)^{\mathrm{T}} \tilde{H}(t)^{\mathrm{T}} Q e_{i}^{2}(t)+e_{i}^{2}(t)^{\mathrm{T}} Q\left(f^{2}\left(x_{i}^{2}(t)\right)-f^{2}\left(s^{2}(t)\right)\right)
\end{aligned}
$$




$$
\begin{aligned}
& +\sum_{i=1}^{N}\left\{\left[c e_{i}^{2}(t)^{\mathrm{T}} \hat{H}(t)^{\mathrm{T}} P e_{i}^{1}(t)-c e_{i}^{1}(t)^{\mathrm{T}} \hat{H}(t)^{\mathrm{T}} P e_{i}^{1}(t)+c e_{i}^{1}(t)^{\mathrm{T}} P \hat{H}(t) e_{i}^{2}(t)\right.\right. \\
& \left.-c e_{i}^{1}(t)^{\mathrm{T}} P \hat{H}(t) e_{i}^{1}(t)\right] d t+\left[c e_{i}^{1}(t)^{\mathrm{T}} \tilde{H}(t)^{\mathrm{T}} Q e_{i}^{2}(t)-c e_{i}^{2}(t)^{\mathrm{T}} \tilde{H}(t)^{\mathrm{T}} Q e_{i}^{2}(t)\right. \\
& \left.+c e_{i}^{2}(t)^{\mathrm{T}} Q \tilde{H}(t) e_{i}^{1}(t)-c e_{i}^{2}(t)^{\mathrm{T}} Q \tilde{H}(t) e_{i}^{2}(t)\right] d t \\
& \left.-\left[2 d e_{i}^{1}(t)^{\mathrm{T}} P e_{i}^{1}(t)+2 d e_{i}^{2}(t)^{\mathrm{T}} Q e_{i}^{2}(t)\right] d t\right\} \\
& +\sum_{i=1}^{N}\left\{\left[B^{1}\left(t, e_{i}^{1}(t)\right)^{\mathrm{T}} P B^{1}\left(t, e_{i}^{1}(t)\right)\right] d t+\left[2 e_{i}^{1}(t)^{\mathrm{T}} P B^{1}\left(t, e_{i}^{1}(t)\right)\right] d W(t)\right. \\
& \left.+\left[B^{2}\left(t, e_{i}^{2}(t)\right)^{\mathrm{T}} Q B^{2}\left(t, e_{i}^{2}(t)\right)\right] d t+\left[2 e_{i}^{2}(t)^{\mathrm{T}} Q B^{2}\left(t, e_{i}^{2}(t)\right)\right] d W(t)\right\} .
\end{aligned}
$$

With Assumption 1 and Lemma 1, we have

$$
\begin{aligned}
& \sum_{i=1}^{N}\left\{\left[f^{1}\left(x_{i}^{1}(t)\right)-f^{1}\left(s^{1}(t)\right)\right]^{\mathrm{T}} P e_{i}^{1}(t)+e_{i}^{1}(t)^{\mathrm{T}} P\left[f^{1}\left(x_{i}^{1}(t)\right)-f^{1}\left(s^{1}(t)\right)\right]\right\} \\
& \quad \leq \sum_{i=1}^{N}\left[f^{1}\left(x_{i}^{1}(t)\right)-f^{1}\left(s^{1}(t)\right)\right]^{\mathrm{T}} P\left[f^{1}\left(x_{i}^{1}(t)\right)-f^{1}\left(s^{1}(t)\right)\right]+\sum_{i=1}^{N} e_{i}^{1}(t)^{\mathrm{T}} P e_{i}^{1}(t) \\
& \quad \leq \lambda^{1} \sum_{i=1}^{N}\left\|f^{1}\left(x_{i}^{1}(t)\right)-f^{1}\left(s^{1}(t)\right)\right\|^{2}+\sum_{i=1}^{N} e_{i}^{1}(t)^{\mathrm{T}} P e_{i}^{1}(t) \\
& \quad \leq \lambda^{1} \sum_{i=1}^{N} e_{i}^{1}(t)^{\mathrm{T}} L_{0}^{\mathrm{T}} L_{0} e_{i}^{1}(t)+\sum_{i=1}^{N} e_{i}^{1}(t)^{\mathrm{T}} P e_{i}^{1}(t) .
\end{aligned}
$$

Similarly,

$$
\begin{aligned}
& \sum_{i=1}^{N}\left\{\left[f^{2}\left(x_{i}^{2}(t)\right)-f^{2}\left(s^{2}(t)\right)\right]^{\mathrm{T}} Q e_{i}^{2}(t)+e_{i}^{2}(t)^{\mathrm{T}} Q\left[f^{2}\left(x_{i}^{2}(t)\right)-f^{2}\left(s^{2}(t)\right)\right]\right\} \\
& \quad \leq \lambda^{2} \sum_{i=1}^{N} e_{i}^{2}(t)^{\mathrm{T}} L_{1}^{\mathrm{T}} L_{1} e_{i}^{2}(t)+\sum_{i=1}^{N} e_{i}^{2}(t)^{\mathrm{T}} Q e_{i}^{2}(t)
\end{aligned}
$$

Furthermore, using Assumption 2 and Lemma 1, we have

$$
\begin{aligned}
& d^{1} \sum_{j=1}^{N} A_{i j}^{1} e_{j}^{1}(t)^{\mathrm{T}} \hat{H}(t)^{\mathrm{T}} P e_{i}^{1}(t)+d^{1} \sum_{j=1}^{N} A_{i j}^{1} e_{i}^{1}(t)^{\mathrm{T}} P \hat{H}(t) e_{j}^{1}(t) \\
& \leq d^{1} \sum_{j=1}^{N}\left|A_{i j}^{1}\right|\left[e_{j}^{1}(t)^{\mathrm{T}} \hat{H}^{\mathrm{T}} P e_{i}^{1}(t)+e_{i}^{1}(t)^{\mathrm{T}} P \hat{H} e_{j}^{1}(t)\right] \\
& \leq d^{1} \sum_{j=1}^{N}\left|A_{i j}^{1}\right|\left[e_{j}^{1}(t)^{\mathrm{T}} e_{j}^{1}(t)+e_{i}^{1}(t) P \hat{H} \hat{H}^{\mathrm{T}} P e_{i}^{1}(t)\right] \\
& \quad=d^{1} \sum_{j=1}^{N}\left|A_{j i}^{1}\right| e_{i}^{1}(t)^{\mathrm{T}} e_{i}^{1}(t)+d^{1} \sum_{j=1}^{N}\left|A_{i j}^{1}\right| e_{i}^{1}(t)^{\mathrm{T}} P \hat{H} \hat{H}^{\mathrm{T}} P e_{i}^{1}(t) .
\end{aligned}
$$


Using the same method, we have

$$
\begin{aligned}
& d^{2} \sum_{j=1}^{N} A_{i j}^{2} e_{j}^{2}(t)^{\mathrm{T}} \tilde{H}(t)^{\mathrm{T}} Q e_{i}^{2}(t)+d^{2} \sum_{j=1}^{N} A_{i j}^{2} e_{i}^{2}(t)^{\mathrm{T}} Q \tilde{H}(t) e_{j}^{2}(t) \\
& \quad \leq d^{2} \sum_{j=1}^{N}\left|A_{j i}^{2}\right| e_{i}^{2}(t)^{\mathrm{T}} e_{i}^{2}(t)+d^{2} \sum_{j=1}^{N}\left|A_{i j}^{2}\right| e_{i}^{2}(t)^{\mathrm{T}} Q \tilde{H} \tilde{H}^{\mathrm{T}} Q e_{i}^{2}(t) .
\end{aligned}
$$

With Assumption 3, we have

$$
\sum_{i=1}^{N} B^{1}\left(t, e_{i}^{1}(t)\right)^{\mathrm{T}} P B^{1}\left(t, e_{i}^{1}(t)\right) \leq \sum_{i=1}^{N} \lambda^{1} B^{1}\left(t, e_{i}^{1}(t)\right)^{\mathrm{T}} B^{1}\left(t, e_{i}^{1}(t)\right) \leq \lambda^{1} \sum_{i=1}^{N} e_{i}^{1}(t)^{\mathrm{T}} L_{2}^{\mathrm{T}} L_{2} e_{i}^{1}(t)
$$

and

$$
\sum_{i=1}^{N} B^{2}\left(t, e_{i}^{2}(t)\right)^{\mathrm{T}} Q B^{2}\left(t, e_{i}^{2}(t)\right) \leq \lambda^{2} \sum_{i=1}^{N} e_{i}^{2}(t)^{\mathrm{T}} L_{3}^{\mathrm{T}} L_{3} e_{i}^{2}(t)
$$

Therefore, according to the discussion mentioned above, equality (15) is

$$
\begin{aligned}
d V(t) \leq & \sum_{i=1}^{N}\left\{\left[e _ { i } ^ { 1 } ( t ) ^ { \mathrm { T } } \left(\lambda^{1} L_{0}^{\mathrm{T}} L_{0}+P+d^{1} \sum_{j=1}^{N}\left|A_{j i}^{1}\right| I+\lambda^{1} L_{2}^{\mathrm{T}} L_{2}-2 d P\right.\right.\right. \\
& \left.\left.+d^{1} \sum_{j=1}^{N}\left|A_{i j}^{1}\right| P \hat{H} \hat{H}^{\mathrm{T}} P-c \hat{H}^{\mathrm{T}} P-c P \hat{H}\right) e_{i}^{1}(t)\right] d t \\
& +e_{i}^{2}(t)^{\mathrm{T}}\left(\lambda^{2} L_{1}^{\mathrm{T}} L_{1}+Q+d^{2} \sum_{j=1}^{N}\left|A_{j i}^{2}\right| I+\lambda^{2} L_{3}^{\mathrm{T}} L_{3}-2 d Q\right. \\
& \left.\left.\left.+d^{2} \sum_{j=1}^{N}\left|A_{i j}^{2}\right| Q \tilde{H} \tilde{H}^{\mathrm{T}} Q-c \tilde{H}^{\mathrm{T}} Q-c Q \tilde{H}\right) e_{i}^{2}(t)\right] d t\right\} \\
& +\sum_{i=1}^{N}\left\{\left[e_{i}^{2}(t)^{\mathrm{T}}\left(c \hat{H}^{\mathrm{T}} P+c Q \tilde{H}\right) e_{i}^{1}(t)\right] d t+\left[e_{i}^{1}(t)^{\mathrm{T}}\left(c P \hat{H}+c \tilde{H}^{\mathrm{T}} Q\right) e_{i}^{2}(t)\right] d t\right\} \\
& +\sum_{i=1}^{N}\left\{\left[2 e_{i}^{1}(t)^{\mathrm{T}} P B^{1}\left(t, e_{i}^{1}(t)\right)\right] d W(t)+\left[2 e_{i}^{2}(t)^{\mathrm{T}} Q B^{2}\left(t, e_{i}^{2}(t)\right)\right] d W(t)\right\}
\end{aligned}
$$

in which

$$
\begin{aligned}
& T_{i}^{1}=\lambda^{1} L_{0}^{\mathrm{T}} L_{0}+P+d^{1} \sum_{j=1}^{N}\left|A_{j i}^{1}\right| I+\lambda^{1} L_{2}^{\mathrm{T}} L_{2}+d^{1} \sum_{j=1}^{N}\left|A_{i j}^{1}\right| P \hat{H} \hat{H}^{\mathrm{T}} P-c \hat{H}^{\mathrm{T}} P-c P \hat{H}-2 d P, \\
& T_{i}^{2}=\lambda^{2} L_{1}^{\mathrm{T}} L_{1}+Q+d^{2} \sum_{j=1}^{N}\left|A_{j i}^{2}\right| I+d^{2} \sum_{j=1}^{N}\left|A_{i j}^{2}\right| Q \tilde{H} \tilde{H}^{\mathrm{T}} Q+\lambda^{2} L_{3}^{\mathrm{T}} L_{3}-c \tilde{H}^{\mathrm{T}} Q-c Q \tilde{H}-2 d Q
\end{aligned}
$$

and

$$
\Phi=c P \hat{H}+c \tilde{H}^{\mathrm{T}} Q .
$$


According to stochastic Lyapunov stability theory, we can guarantee the mean-square heterogeneous asymptotical synchronization of interdependent networks (1) under the adaptive control law (11) if

$$
\left[\begin{array}{cc}
T_{i}^{1} & \Phi \\
\Phi^{\mathrm{T}} & T_{i}^{2}
\end{array}\right]<0 .
$$

At the same time, it should be noted that the inequality (17) is not the standard linear matrix inequality, it can be written equivalently as inequality (10) with Lemma 2.

Thus, the proof is completed.

The discussions above tell us that if there exist two positive matrices $P$ and $Q$ satisfying LMI (10), then the interdependent networks (1) with stochastic disturbances reach mean-square heterogeneous asymptotical synchronization. When there are no stochastic disturbances, with similar methods to the proof of Theorem 1, Corollary 1 leads to the interdependent networks (1) to adaptive heterogeneous synchronization.

Corollary 1 Suppose Assumptions 1-3 hold. If there exist two positive matrices $P$ and $Q$, such that

$$
\Lambda=\left[\begin{array}{cccc}
\tilde{T}_{i}^{1} & \Phi & \Theta^{1} & 0 \\
\Phi^{\mathrm{T}} & \tilde{T}_{i}^{2} & 0 & \Theta^{2} \\
\Theta^{1^{\mathrm{T}}} & 0 & \Psi^{1} & 0 \\
0 & \Theta^{2^{\mathrm{T}}} & 0 & \Psi^{2}
\end{array}\right]<0,
$$

where

$$
\begin{aligned}
& \tilde{T}_{i}^{1}=\lambda^{1} L_{0}^{\mathrm{T}} L_{0}+P+d^{1} \sum_{j=1}^{N}\left|A_{j i}^{1}\right| I-c \hat{H}^{\mathrm{T}} P-c P \hat{H}-2 d P, \\
& \tilde{T}_{i}^{2}=\lambda^{2} L_{1}^{\mathrm{T}} L_{1}+Q+d^{2} \sum_{j=1}^{N}\left|A_{j i}^{2}\right| I-c \tilde{H}^{\mathrm{T}} Q-c Q \tilde{H}-2 d Q, \\
& \Phi=c P \hat{H}+c \tilde{H}^{\mathrm{T}} Q, \quad \Theta^{1}=P \hat{H}, \quad \Theta^{2}=Q \tilde{H}, \\
& \Psi^{1}=\frac{-1}{d^{1} \sum_{j=1}^{N}\left|A_{i j}^{1}\right|} I, \quad \Psi^{2}=\frac{-1}{d^{2} \sum_{j=1}^{N}\left|A_{i j}^{2}\right|} I,
\end{aligned}
$$

then the interdependent networks (1) without stochastic disturbances reach adaptive heterogeneous synchronization under the control law (11) and the adaptive law (12).

\section{Simulations}

In this section, some examples are shown to verify the feasibility and effectiveness of the conditions presented in Sect. 3. The sub-network $G^{1}$ is a BA network which consists of 100 nodes, where the average degree is 6.0800 , the average path length is 2.5440 and the clustering coefficient is 0.2094 . The sub-network $G^{2}$ is a SW network which has the same nodes. Among them, the average degree is 10.4000 , the average path length is 2.4180 and the clustering coefficient is 0.4191 . All the simulations take the Rössler system and Lü 
system as the dynamical system of the nodes in the sub-network $G^{1}$ and the sub-network $G^{2}$, separately. The Rössler system can be described by [29]

$$
\left\{\begin{array}{l}
\dot{x}_{i 1}=-\left(x_{i 2}+x_{i 3}\right), \\
\dot{x}_{i 2}=x_{i 1}+a_{1} x_{i 2}, \\
\dot{x}_{i 3}=a_{2}+x_{i 3}\left(x_{i 1}-a_{3}\right),
\end{array} \quad i=1,2, \ldots, 100,\right.
$$

where $a_{1}, a_{2}, a_{3}$ are real constants. While $a_{1}=0.5, a_{2}=0.2, a_{3}=5.7$, the Rössler system is chaotic. At the same time, the Lü system can be described by [29]

$$
\left\{\begin{array}{l}
\dot{y}_{i 1}=b_{1}\left(y_{i 2}-y_{i 1}\right) \\
\dot{y}_{i 2}=-y_{i 1} y_{i 3}+b_{2} y_{i 2}, \quad i=1,2, \ldots, 100 . \\
\dot{y}_{i 3}=y_{i 1} y_{i 2}-b_{3} y_{i 3}
\end{array}\right.
$$

When $b_{1}=36, b_{2}=20, b_{3}=3$, the Lü system is chaotic.

Let the coupling functions be as follows:

$$
H\left(x_{i}(t)\right)=\left(-x_{i 1}(t), x_{i 2}(t),\left(x_{i 3}(t)\right)^{2}\right),
$$

and

$$
H\left(y_{i}(t)\right)=\left(-y_{i 1}(t), y_{i 2}(t),\left(y_{i 3}(t)\right)^{2}\right) .
$$

In addition, we let

$$
B\left(x_{i}(t)\right)=\left(\sin \left(x_{i 1}(t)-s_{1}^{1}(t)\right), x_{i 2}(t)-s_{2}^{1}(t), \sin \left(x_{i 3}(t)-s_{3}^{1}(t)\right)\right),
$$

and

$$
B\left(y_{i}(t)\right)=\left(\sin \left(y_{i 1}(t)-s_{1}^{2}(t)\right), y_{i 2}(t)-s_{2}^{2}(t), \sin \left(y_{i 3}(t)-s_{3}^{2}(t)\right)\right) .
$$

Furthermore, we assume $c=0.26, d^{1}=d^{2}=0.8$, adaptive law initial values $d_{i}^{1}=0.04 * i$, $d_{i}^{2}=1.5 * i$, and feedback gains $k_{i}^{1}=k_{i}^{2}=1$. The initial values of the isolated systems are $l^{1}=(5,-8,2)$ and $l^{2}=(-6,8,5)$, respectively. The initial values of sub-network $G^{1}$ and $G^{2}$ are $x_{i}=(-0.01 * i, 0.1 * i, 0.5 * i)$ and $y_{i}=(-2 * i, 0.05 * i, 0.15 * i), i=1,2, \ldots, 100$, separately.

Figures 1 and 2 display the trajectories of sub-network $G^{1}$ and sub-network $G^{2}$ without controllers. The trajectories of sub-network $G^{1}$ and sub-network $G^{2}$ nodes are chaotic or uncontrollable with time. According to LMI (10), using LMI Control Toolbox, there exist the following positive matrices $P$ and $Q$ satisfying the condition (10):

$$
P=\left[\begin{array}{lll}
1.3811 & 0.0014 & 0.4292 \\
0.0014 & 0.5315 & 0.0242 \\
0.4292 & 0.0242 & 0.4677
\end{array}\right], \quad Q=\left[\begin{array}{lll}
3.4637 & 0.0962 & 0.6452 \\
0.0962 & 2.0463 & 0.2969 \\
0.6452 & 0.2969 & 0.7271
\end{array}\right] \text {. }
$$

With the suitable controllers (11), the trajectories of the error systems tend to zero quickly in Figs. 3 and 4. They tell us that the interdependent networks (1) have reached 

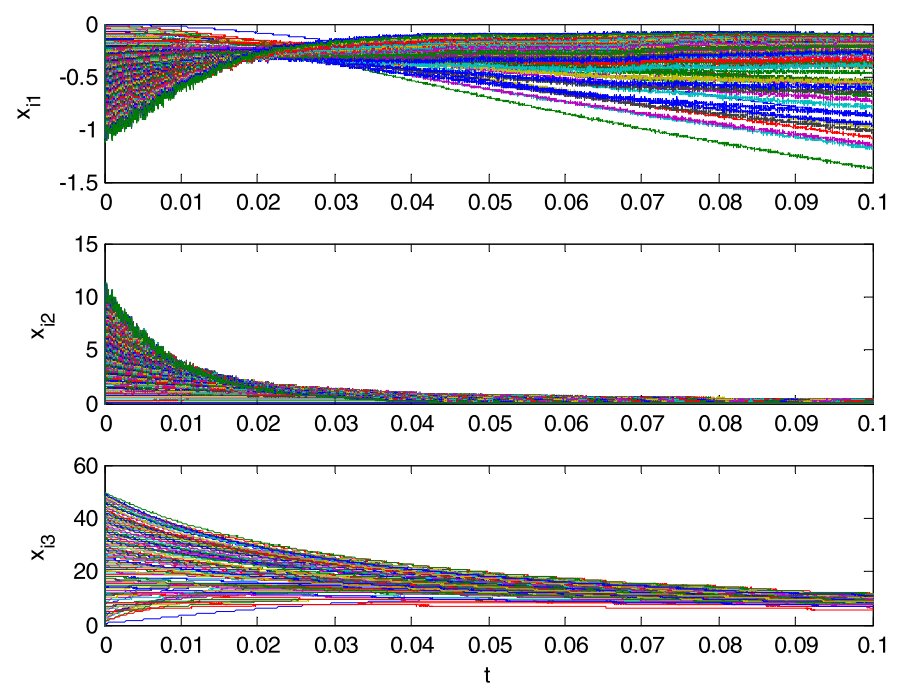

Figure 1 Trajectories of $G^{1}$ without controller
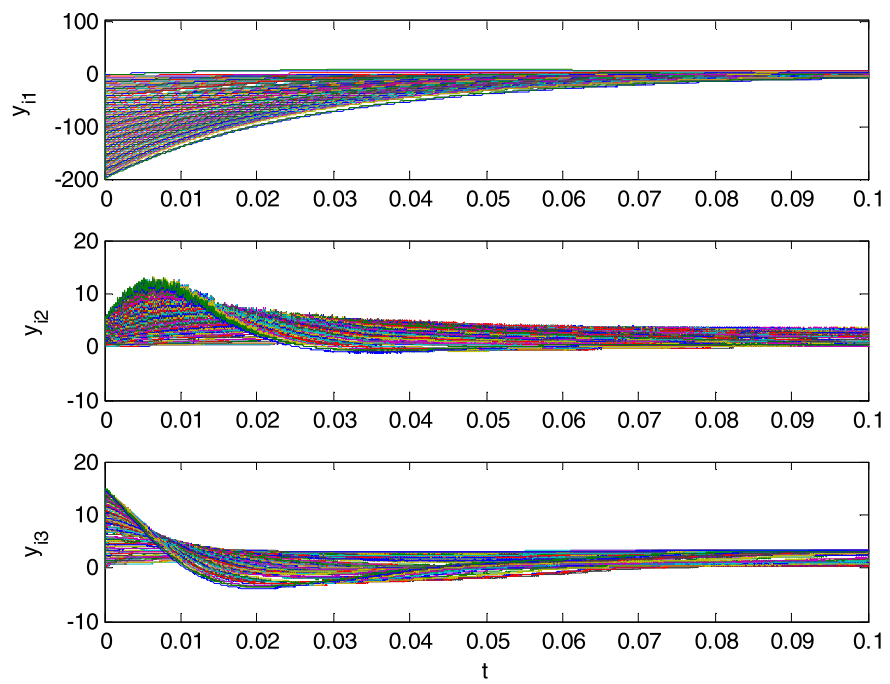

Figure 2 Trajectories of $G^{2}$ without controller

its mean-square heterogeneous asymptotical synchronization. It is worth noting that the adaptive law also reaches the stable values while the error systems are asymptotically stable at the origin (see Figs. 5 and 6). They show the feasibility and effectiveness of the conditions provided in this paper.

\section{Conclusions}

In the real world, many critical infrastructures or systems interact with and depend on each other. Recently, protecting and improving such critical infrastructures or systems using network science has become more and more important. In this paper, we mainly investigate the mean-square heterogeneous asymptotical synchronization of interdependent networks with stochastic disturbances. Compared with Refs. [19-22, 30], our net- 

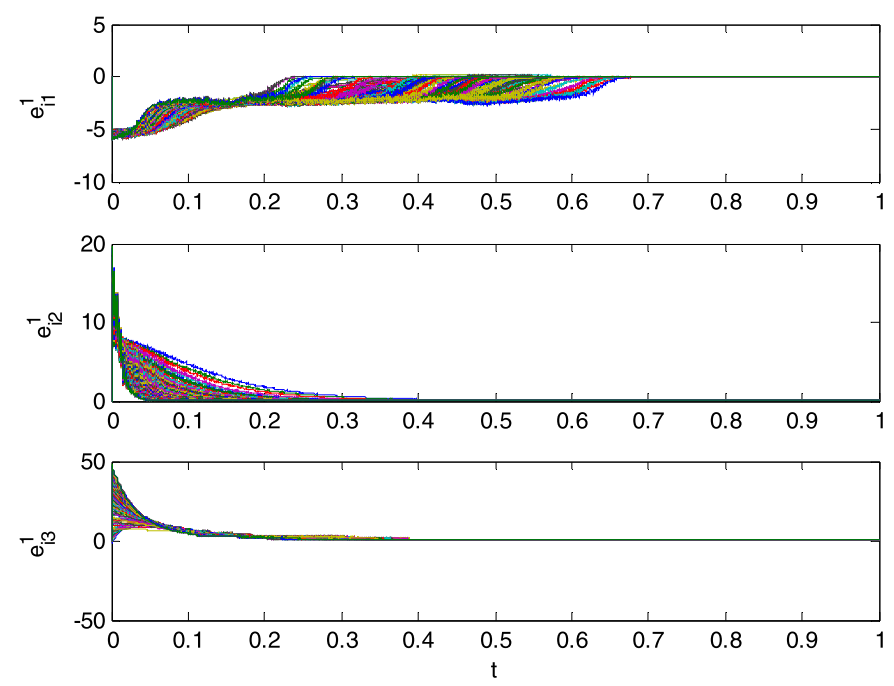

Figure 3 Trajectories of the error system (8) about $G^{1}$
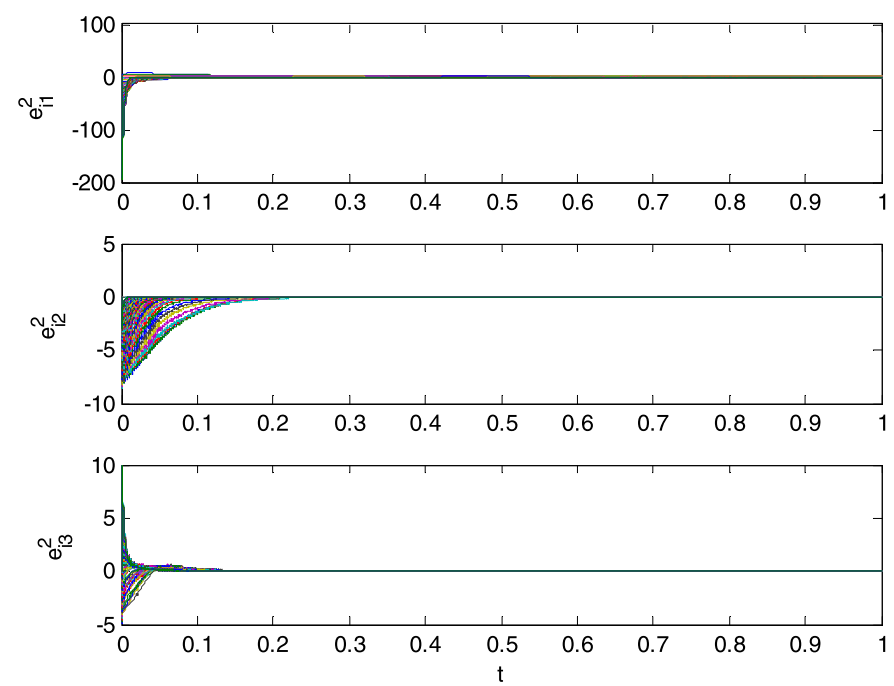

Figure 4 Trajectories of the error system (8) about $G^{2}$

works not only have the intra-coupling connections but also have the inter-coupling dependence with each other. Compared with Refs. $[13,14]$, stochastic disturbances exist our networks. Based on the stochastic Lyapunov stability theory, adaptive control, the Itô formula and the linear matrix inequality, some sufficient conditions are given to ensure the mean-square heterogeneous asymptotical synchronization for the interdependent networks. Finally, some illustrative examples are given to verify the accuracy and effectiveness of the proposed theory. Simulations show that the given results in this paper perform very well. The results and understandings of this paper can be instructive for protecting or building more realistic interdependent networks, such as electric-calculator networks and aviation-railway-road networks with stochastic disturbances. It also can be used to deal with the problems of networks of networks. 


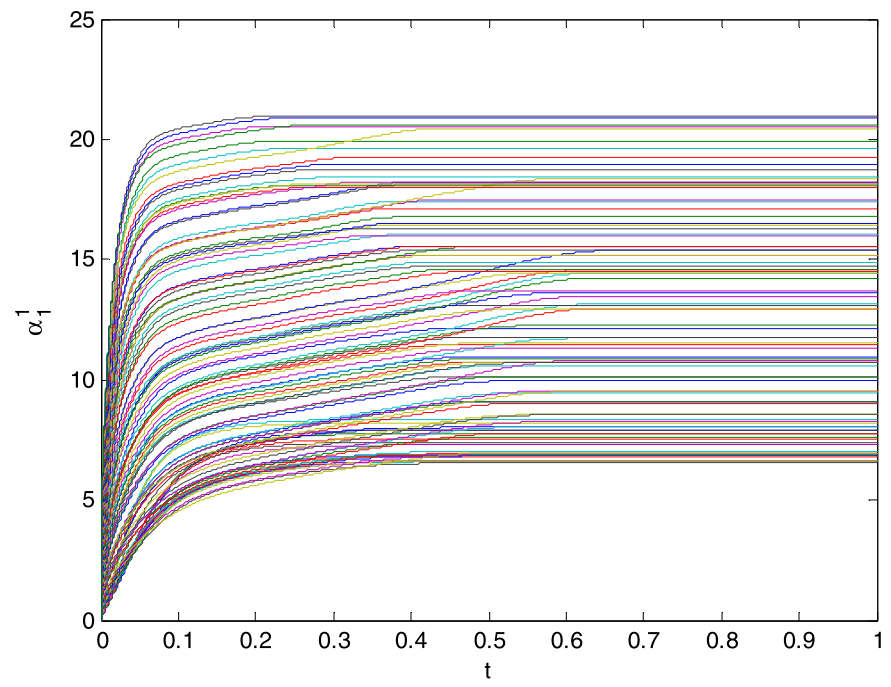

Figure 5 Trajectories of the adaptive law (12) about $G^{1}$

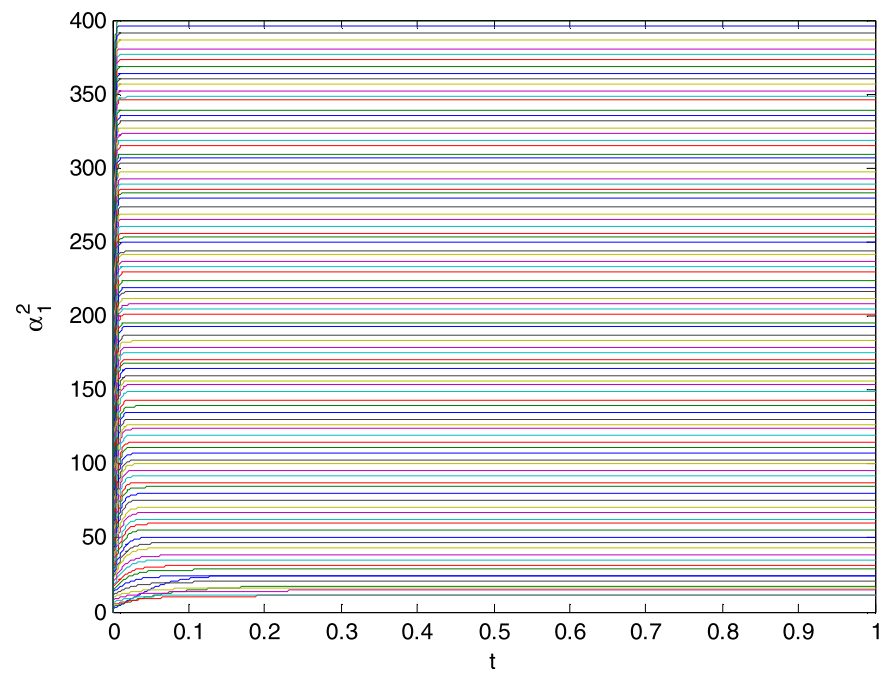

Figure 6 Trajectories of the adaptive law (12) about $G^{2}$

\section{Acknowledgements}

The authors would like to thank the editors and the anonymous reviewers for their useful comments and suggestions.

Funding

This work is supported by the National Natural Science Foundation of China under Grant 61473338.

\section{Competing interests}

The authors declare that they have no competing interests.

\section{Authors' contributions}

All authors contributed equally and significantly in this manuscript, and they read and approved the final manuscript.

\section{Publisher's Note}

Springer Nature remains neutral with regard to jurisdictional claims in published maps and institutional affiliations.

Received: 14 March 2019 Accepted: 24 April 2019 Published online: 22 May 2019 


\section{References}

1. Stasiuk, A.I., Hryshchuk, R.V., Goncharova, L.L.: A mathematical cybersecurity model of a computer network for the control of power supply of traction substations. Cybern. Syst. 53(3), 476-484 (2017)

2. Cardillo, A., Zanin, M., Gomez-Gardenes, J., Romance, M., del Amo, A.J.G., Boccaletti, S.: Modeling the multi-layer nature of the European air transport network: resilience and passengers re-scheduling under random failures. Eur. Phys. J. Spec. Top. 215(1), 23-33 (2013)

3. Rosato, V., Issacharoff, L., Tiriticco, F., Meloni, S., Porcellinis, S.D., Setola, R.: Modelling interdependent infrastructures using interacting dynamical models. Int. J. Crit. Infrastructures 4(1), 63-79 (2008)

4. Buldyrev, S.V., Parshani, R., Paul, G., Stanley, H.E., Havlin, S.: Catastrophic cascade of failures in interdependent networks. Nature 464(7291), 1025-1028 (2010)

5. Gao, J.X., Buldyrev, S.V., Stanley, H.E., Havlin, S.: Networks formed from interdependent networks. Nat. Phys. 8(1), 40-48 (2011)

6. Zhang, P., Cheng, B.S., Zhao, Z., Li, D.Q., Lu, G.Q., Wang, Y.P., Xiao, J.H.: The robustness of interdependent transportation networks under targeted attack. EPL 103, 68005 (2013)

7. Ji, X.P., Wang, B., Liu, D.C., Chen, G., Tang, F., Wei, D.Q., Tu, L.: Improving interdependent networks robustness by adding connectivity links. Physica A 444, 9-19 (2016)

8. Xu, Q., Zhuang, S.X., Hu, D., Zeng, Y.F., Xiao, J.: Generalized mutual synchronization between two controlled interdependent networks. Abstr. Appl. Anal. 2014, 453149 (2014)

9. Wang, Y.J., Tu, L.L., Li, K.Y., Song, S.: Local adaptive heterogeneous synchronization for interdependent networks with delayed coupling. Acta Phys. Sin. 67(5), 050504 (2018)

10. Shen, A., Guo, J.L., Wang, Z.J.: Research on methods for improving robustness of cascading failures of interdependent networks. Wirel. Pers. Commun. 95(3), 2111-2126 (2017)

11. Wang, Z., Szolnoki, A., Perc, M.: Interdependent network reciprocity in evolutionary games. Sci. Rep. 3, 1183 (2013)

12. Wang, Z., Szolnoki, A., Perc, M.: Self-organization towards optimally interdependent networks by means of coevolution. New J. Phys. 16, 033041 (2014)

13. Wang, Z.Q., Xu, Y., Yang, H.: Levy noise induced stochastic resonance in an FHN model. Sci. China, Technol. Sci. 59(3), $371-375(2016)$

14. Wang, Y., Lai, Y.C., Zheng, Z.G.: Route to noise-induced synchronization in an ensemble of uncoupled chaotic systems. Phys. Rev. E 81(3), 036201 (2010)

15. Nagai, K.H., Hiroshi, K.: Noise-induced synchronization of a large population of globally coupled nonidentical oscillators. Phys. Rev. E 81(6), 065202 (2010)

16. Liu, Y.J., Guo, B.Z., Park, J.H., Lee, S.M.: Nonfragile exponential synchronization of delayed complex dynamical networks with memory sampled-data control. IEEE Trans. Neural Netw. Learn. Syst. 29(1), 118-128 (2018)

17. Yu, R., Zhang, H.G., Wang, Z.L., Liu, Y.: Synchronization criterion of complex networks with time-delay under mixed topologies. Neurocomputing 295, 8-16 (2018)

18. Yang, X.S., Lam, J., Ho, D.W.C., Feng, Z.G.: Fixed-time synchronization of complex networks with impulsive effects via non-chattering control. IEEE Trans. Autom. Control 62(11), 5511-5521 (2017)

19. Shi, X.R., Wang, Z.L., Han, L.X.: Finite-time stochastic synchronization of time-delay neural networks with noise disturbance. Nonlinear Dyn. 88(4), 2747-2755 (2017)

20. Yang, X.S., Cao, J.D.: Finite-time stochastic synchronization of complex networks. Appl. Math. Model. 34(11), $3631-3641(2010)$

21. Xu, Y.H., Zhou, W.N., Fang, J.A., Sun, W., Pan, L.: Adaptive synchronization of stochastic time-varying delay dynamical networks with complex-variable systems. Nonlinear Dyn. 81(4), 1717-1726 (2015)

22. Li, L.L., Jian, J.G.: Finite-time synchronization of chaotic complex networks with stochastic disturbance. Entropy 17(1), 39-51 (2015)

23. Liu, X., Ho, D.W.C., Song, Q., Gao, J.: Finite/fixed-time pinning synchronization of complex networks with stochastic disturbances. IEEE Trans. Cybern. 49 2398-2403 (2018)

24. Wei, X., Wu, X.Q., Lu, J.A., Zhao, J.C.: Counterpart synchronization of duplex networks with delayed nodes and noise perturbation. J. Stat. Mech. Theory Exp. 2015, 11021 (2015)

25. Zhao, X.Y., Zhou, J., Lu, J.A.: Pinning synchronization of multiplex delayed networks with stochastic perturbations. IEEE Trans. Cybern. 2018, $2861822(2018)$

26. Um, J., Minnhagen, P., Kim, B.J.: Synchronization in interdependent networks. Chaos 21(2), 025106 (2011)

27. Tu, L.L., Liu, H.F., Yu, L.: Local adaptive H infinity consistency of delayed complex networks with noise. Acta Phys. Sin. 62(14), 140506 (2013)

28. Boyd, S., Ghaoui, L.E., Feron, E., Balakrishnan, V.: Linear matrix inequalities in system and control theory, pp. 7-12. Society for Industrial and Applied Mathematics (1994)

29. Lu, J.A., Liu, H., Chen, J.: Synchronization in complex dynamical networks, pp. 31-35. Higher Education Press, Beijing (2016)

30. Wu, X.Q., Zhou, C.S., Chen, G.R., Lu, J.A.: Detecting the topologies of complex networks with stochastic perturbations. Chaos 21(4), 043129 (2011) 